\title{
Environment-controlled Floquet-state paramagnetism
}

\author{
Onno R. Diermann, ${ }^{1}$ Heinz-Jürgen Schmidt, ${ }^{2}$ Jürgen Schnack, ${ }^{3}$ and Martin Holthaus $\circledast^{1}$ \\ ${ }^{1}$ Carl von Ossietzky Universität, Institut für Physik, D-26111 Oldenburg, Germany \\ ${ }^{2}$ Universität Osnabrück, Fachbereich Physik, D-49069 Osnabrück, Germany \\ ${ }^{3}$ Universität Bielefeld, Fakultät für Physik, D-33501 Bielefeld, Germany
}

(Received 22 March 2020; accepted 19 May 2020; published 5 June 2020)

\begin{abstract}
We study the response of ideal spin systems which are interacting with both a strong oscillating magnetic field, and a thermal environment, to a weak probing magnetic field. We demonstrate that even the sign of the resulting mean magnetization depends on the amplitude of the driving field and that its absolute value can be significantly larger than the equilibrium magnetization in the absence of time-periodic forcing. Since the underlying Floquet-state occupation probabilities are determined by the precise form of the system-bath coupling, future measurements of such effects have the potential to establish a particularly innovative line of research, providing information on nonequilibrium thermodynamics and giving access to quantities which usually remain hidden when probing equilibrium systems.
\end{abstract}

DOI: 10.1103/PhysRevResearch.2.023293

\section{INTRODUCTION}

The subject of quantum thermal magnetism, i.e., the behavior of quantized magnetic moments simultaneously affected by both an external static magnetic field and a thermal environment, has been addressed by Brillouin already in 1927 $[1,2]$ : the magnetic moment $\mu$ of atoms possessing an electron shell with total angular momentum $J$ and Landé $g$-factor $g_{J}$ takes the form $\mu=g_{J} \mu_{\mathrm{B}} J$, where $\mu_{\mathrm{B}}$ is the Bohr magneton. When such magnetic moments are exposed to a homogeneous, constant magnetic field of strength $B_{0}$, quantization of angular momentum gives rise to the energy levels

$$
E_{m}=-m g_{J} \mu_{\mathrm{B}} B_{0},
$$

with $m=-J, \ldots, J$ denoting the magnetic quantum number. Assuming that the atoms are interacting with a surrounding of temperature $T$, these levels are occupied according to the universal Boltzmann distribution

$$
p_{m}^{\mathrm{eq}}=\frac{1}{Z_{0}} \exp \left(-\frac{E_{m}}{k_{\mathrm{B}} T}\right),
$$

where $k_{\mathrm{B}}$ is Boltzmann's constant, normalization is ensured by the familiar canonical partition function $Z_{0}=$ $\sum_{m=-J}^{J} \exp \left(-E_{m} / k_{\mathrm{B}} T\right)$, and the superscript "eq" indicates thermal equilibrium. With $N$ magnetic moments in a volume $V$ the resulting magnetization reads

$$
M=\frac{N}{V} g_{J} \mu_{\mathrm{B}}\langle m\rangle_{\mathrm{eq}},
$$

Published by the American Physical Society under the terms of the Creative Commons Attribution 4.0 International license. Further distribution of this work must maintain attribution to the author(s) and the published article's title, journal citation, and DOI. where the thermal expectation value

$$
\langle m\rangle_{\mathrm{eq}}=\sum_{m=-J}^{J} m p_{m}^{\mathrm{eq}}
$$

can be expressed explicitly in terms of the so-called Brillouin function of order $J$ [2]. An experimental study of such spin paramagnetism with $\mathrm{Cr}^{+++}, \mathrm{Fe}^{+++}$, and $\mathrm{Gd}^{+++}$by Henry in 1952 has resulted in spectacular agreement with this theoretical prediction [3]. Thus, when accepting equilibrium thermodynamics as a firmly established proposition, measurement of the thermal magnetization of paramagnetic substances provides a striking experimental proof for the space quantization of magnetic dipoles [3].

The present deliberations are meant to demonstrate that this time-honored reasoning can be logically reversed by novel experiments involving oscillating fields which may be so strong that they fall outside the regime of linear response, and therefore are not covered by the well-established concept of ac susceptibility [4-6]: accepting the quantization of angular momentum as given fact, measurement of the mean magnetization of paramagnetic materials in strong time-periodic magnetic fields provides information on a particular form of nonequilibrium thermodynamics that is becoming known as "periodic thermodynamics" [7]. Such periodic thermodynamics refers to quantum systems that are both driven by a time-periodic external force and interacting with a heat bath of fixed temperature [8-21]. As a consequence of time-periodic forcing, the driven system possesses a complete set of Floquet states; as a consequence of their interaction with the heat bath, a nonequilibrium steady state establishes itself, which is characterized by a quasistationary distribution of Floquet-state occupation probabilities. Quite unlike the universal Boltzmann distribution (2), such Floquet-state distributions depend on the very details of the system-bath interaction [7,22-24]. Therefore, measurements of these distributions, or of observables directly governed by them, provide experimental access to 
system-bath coupling mechanisms, or to details of the heat bath itself, which remain unobservable when probing equilibrium systems. Here we outline the ramifications of this general concept for driven spin systems, extending our previous work [25]. We start with a brief sketch of the theoretical basics in Sec. II, then discuss numerical model calculations providing cases in point in Sec. III, and summarize the key issues in Sec. IV.

\section{CONCEPT OF QUASITHERMAL MAGNETISM}

We write the Hamiltonian of a spin $J$ acted on by both a static and an oscillating magnetic field in the form

$$
H(t)=H_{0}+H_{\mathrm{osc}}(t),
$$

where

$$
H_{0}=\hbar \omega_{0} S_{z},
$$

with $S_{x}, S_{y}, S_{z}$ denoting the components of the dimensionless spin operator, so that the frequency $\omega_{0}=-g_{J} \mu_{\mathrm{B}} B_{0} / \hbar$ encodes the strength of the static field $B_{0}$ oriented along the $z$ axis, in accordance with Eq. (1). Note that $\omega_{0}$ may adopt both signs, depending on the sign of $g_{J}$. Considering, to begin with, an oscillating field which is right-circularly polarized in the $x-y$ plane with angular frequency $\omega$ and amplitude $B_{1}$, one has

$$
H_{\mathrm{osc}}^{(r)}(t)=\hbar F\left[S_{x} \cos (\omega t)+S_{y} \sin (\omega t)\right],
$$

thus introducing a further frequency $F=g_{J} \mu_{\mathrm{B}} B_{1} / \hbar$. The Floquet states

$$
\left|\psi_{m}(t)\right\rangle=\left|u_{m}(t)\right\rangle \exp \left(-i \varepsilon_{m} t / \hbar\right)
$$

of this system, that is, the complete set of solutions of the time-dependent Schrödinger equation pertaining to the Hamiltonian (5) with periodic Floquet functions $\left|u_{m}(t)\right\rangle=$ $\left|u_{m}(t+2 \pi / \omega)\right\rangle$, have been determined analytically [25]. Their quasienergies are given by

$$
\varepsilon_{m}=\left[\frac{\hbar \omega}{2}\right] \pm m \hbar \Omega \quad \bmod \hbar \omega,
$$

where the contribution $\hbar \omega / 2$ in square brackets figures for half-integer $J$ only and vanishes otherwise,

$$
\Omega=\sqrt{\left(\omega_{0}-\omega\right)^{2}+F^{2}}
$$

is the Rabi frequency, and the \pm sign may conventionally be selected such that $\varepsilon_{m}$ reduces to the energy $E_{m}$ (modulo $\hbar \omega$ ) when $F$ goes to zero. Figure 1 depicts an interval $-\hbar \omega / 2 \leqslant$ $\varepsilon<+\hbar \omega / 2$ of this quasienergy spectrum (9), corresponding to one Brillouin zone, for $J=7 / 2$ and $\omega_{0} / \omega=0.1$ vs the scaled driving strength $F / \omega$. In this case of high-frequency driving with positive $\omega_{0}$, that is, for $\omega \gg \omega_{0}>0$, the canonical representatives of the quasienergy eigenvalues, i.e., those representatives which connect continuously to the energy eigenvalues of $H_{0}$, effectively attract each other when the driving amplitude is increased from zero to small finite values, and cross. This complete quasienergy collapse indicates the principal resonance $\Omega=\omega$, implying $(F / \omega)^{2}=2 \omega_{0} / \omega-$ $\left(\omega_{0} / \omega\right)^{2}$, so that all quasienergies are degenerate (modulo $\hbar \omega)$. Also visible in Fig. 1 is another conspicuous resonance

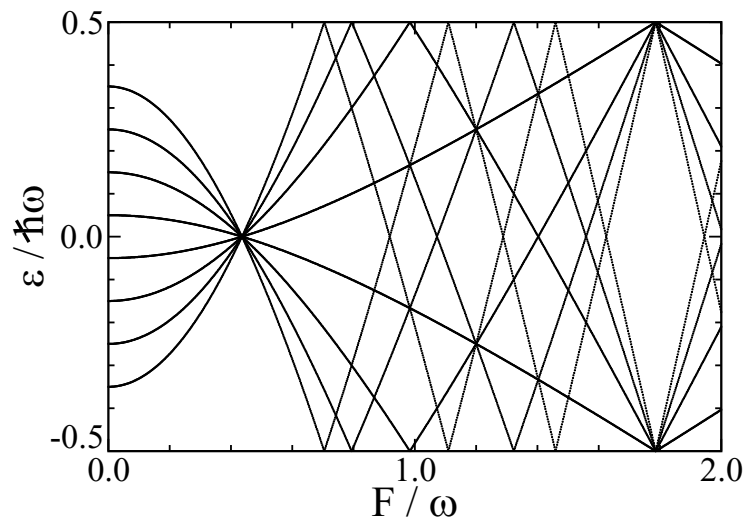

FIG. 1. One Brillouin zone of quasienergies for a spin $J=7 / 2$ driven by a right-circularly polarized high-frequency magnetic field according to Eq. (7), and exposed to a static magnetic field of scaled strength $\omega_{0} / \omega=0.1$. Observe the complete quasienergy collapse at $F / \omega \approx 0.44$, indicating the principal resonance $\Omega / \omega=1$. Further particularly high degeneracies appear at $F / \omega=1.2(\Omega / \omega=3 / 2)$ and $F / \omega \approx 1.79(\Omega / \omega=2)$.

for which $\Omega=2 \omega$, and a further, less obvious resonance occurring when $\Omega=3 \omega / 2$, giving rise to two points of degeneracy in the Brillouin zone, separated by $\hbar \omega / 2$.

The Hamiltonian (5) of the driven spin acts on a Hilbert space $\mathcal{H}_{\text {sys }}$ of finite dimension $2 J+1$. We now couple this system weakly to a heat bath consisting of infinitely many, thermally occupied harmonic oscillators, described by a bath Hamiltonian $H_{\text {bath }}$ on a corresponding Hilbert space $\mathcal{H}_{\text {bath }}$. Let us briefly summarize the required formal steps [22,23,25]: the total Hamiltonian on the composite space $\mathcal{H}_{\text {sys }} \otimes \mathcal{H}_{\text {bath }}$ can be written as [26]

$$
H_{\text {total }}(t)=H(t) \otimes \mathbb{1}+\mathbb{1} \otimes H_{\text {bath }}+H_{\text {int }} .
$$

For the sake of definiteness, here we choose a bilinear systembath coupling of the form

$$
H_{\mathrm{int}}=V \otimes \sum_{\widetilde{\omega}}\left(b_{\widetilde{\omega}}+b_{\widetilde{\omega}}^{\dagger}\right),
$$

where

$$
V=\gamma_{x} S_{x}+\gamma_{y} S_{y}+\gamma_{z} S_{z}
$$

with adjustable coefficients $\gamma_{x}, \gamma_{y}, \gamma_{z}$ carrying the dimension of energy and where $b_{\widetilde{\omega}}\left(b_{\widetilde{\omega}}^{\dagger}\right)$ denotes an annihilation (creation) operator for a bath oscillator with frequency $\widetilde{\omega}$; the sum in Eq. (12) extends over all such oscillators. Naturally, the constraint that the system-bath coupling be weak requires $\left|\gamma_{x, y, z}\right| \ll \hbar\left|\omega_{0}\right|$.

Writing the Fourier decomposition of a transition matrix element between Floquet states $i$ and $f$ as

$$
\left\langle u_{f}(t)|V| u_{i}(t)\right\rangle=\sum_{\ell \in \mathbb{Z}} V_{f i}^{(\ell)} \exp (i \ell \omega t),
$$

and introducing the corresponding transition frequencies

$$
\omega_{f i}^{(\ell)}=\left(\varepsilon_{f}-\varepsilon_{i}\right) / \hbar+\ell \omega,
$$


one finds the partial transition rates

$$
\Gamma_{f i}^{(\ell)}=\frac{2 \pi}{\hbar^{2}}\left|V_{f i}^{(\ell)}\right|^{2} N\left(\omega_{f i}^{(\ell)}\right) \varrho\left(\left|\omega_{f i}^{(\ell)}\right|\right) .
$$

Here the thermal expectation values

$$
N(\widetilde{\omega})=\frac{ \pm 1}{\exp (\beta \hbar \widetilde{\omega})-1}
$$

quantify the mean occupation numbers of the bath oscillators, with $\beta=1 /\left(k_{\mathrm{B}} T\right)$ indicating the inverse bath temperature. The plus (minus) sign applies to positive (negative) transition frequencies $\widetilde{\omega}$; observe that each transition among Floquet states is associated with an infinite ladder (15) of such frequencies. Moreover, the quantity $\varrho(\widetilde{\omega})$ entering the partial rates (16) denotes the spectral density of the bath oscillators. The total rate of transitions among the Floquet states $i$ and $f$ then is given by the sum

$$
\Gamma_{f i}=\sum_{\ell \in \mathbb{Z}} \Gamma_{f i}^{(\ell)} .
$$

The desired quasistationary distribution $\left\{p_{n}\right\}$ of Floquet-state occupation probabilities which results from the combined effects of time-periodic driving and bath-induced transitions, and which in periodic thermodynamics replaces the equilibrium Boltzmann distribution (2), finally is obtained as a solution to the master equation

$$
\sum_{m}\left(\Gamma_{n m} p_{m}-\Gamma_{m n} p_{n}\right)=0 .
$$

The golden rule for Floquet states, which underlies the partial rates (16), requires that the bath is not affected by the timeperiodic driving field [13], in the sense of the usual Born approximation. If this condition is not met, and the bath itself "feels" the drive, one may obtain different results, as has been exemplified in Ref. [8]. The analytical calculation of such Floquet-state occupation probabilities is feasible for certain integrable systems only [13,20,22,23,25]; in general, the above framework has to be implemented numerically.

Thus, when an ideal paramagnetic material is strongly driven by an oscillating field $B_{1}$, while interacting with a thermal environment and being probed by a static field $B_{0}$ directed along the $z$ axis, the resulting nonequilibrium "quasithermal" magnetization is still given by a relation of the general form (3), but the usual thermal expectation value (4) now has to be replaced by the Floquet-state expectation value [25]

$$
\langle\langle m\rangle\rangle=\sum_{m=-J}^{J}\left\langle\left\langle u_{m}(t)\left|S_{z}\right| u_{m}(t)\right\rangle\right\rangle p_{m},
$$

where double brackets indicate one-cycle averaging,

$$
\left\langle\left\langle u_{m}(t)\left|S_{z}\right| u_{m}(t)\right\rangle\right\rangle=\frac{\omega}{2 \pi} \int_{0}^{2 \pi / \omega} d t\left\langle u_{m}(t)\left|S_{z}\right| u_{m}(t)\right\rangle .
$$

As emphasized above, the particular interest in this scenario stems from the observation that, in contrast to their equilibrium antecessors (2), the Floquet-state occupation probabilities $p_{m}$ are not universal, but do depend on the way the driven system is interacting with its environment. We suggest that this unfamiliar, but experimentally detectable fact may be exploited in a twofold manner: measuring Floquet-state
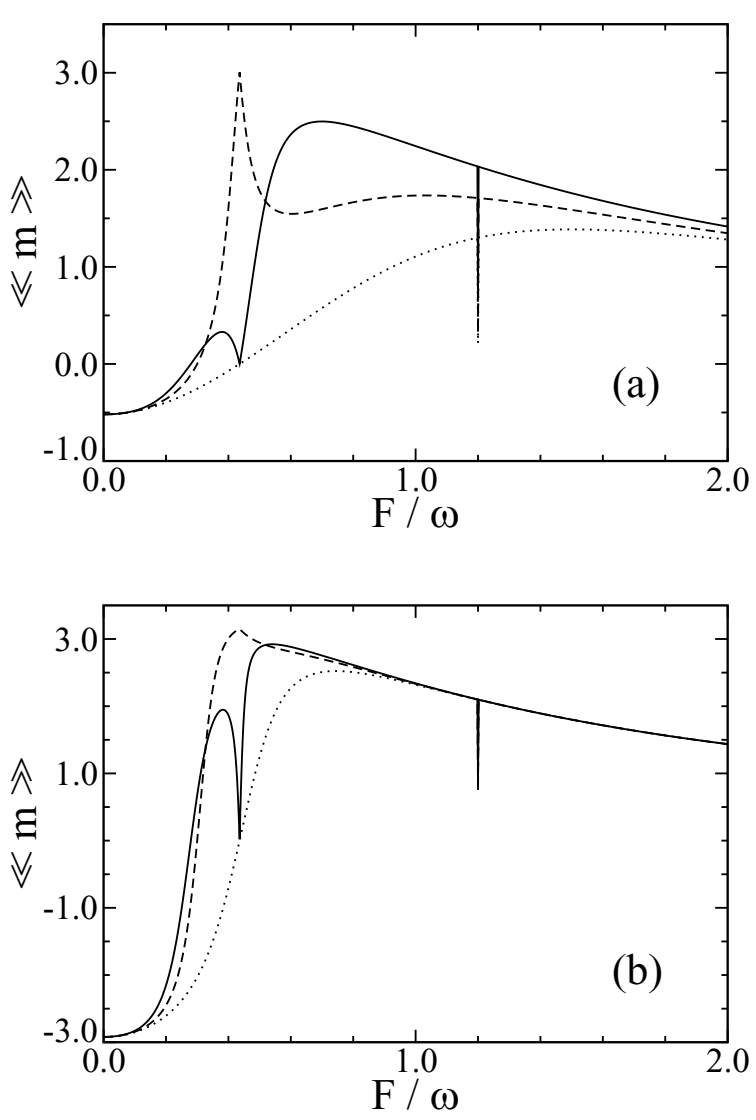

FIG. 2. Quasithermal expectation values (20) for $J=7 / 2$, $\omega_{0} / \omega=0.1$, and right-circularly polarized driving described by Eq. (7). Here $\gamma_{y}=\gamma_{z}=0$; the scaled bath temperature is $k_{\mathrm{B}} T /(\hbar \omega)=1.0$ in the upper panel (a) and $k_{\mathrm{B}} T /(\hbar \omega)=0.1$ in the lower one (b). The bath density of states is taken to be constant (dotted), quadratic (dashed) according to Eq. (22), and Gaussian (full lines) with $\omega_{\mathrm{c}} / \omega=5.0$; see Eq. (23).

expectation values, such as the quasithermal averages (20), provides information on the system-bath coupling, or on properties of the bath. On the other hand, deliberate engineering of the system-bath coupling may allow one to create systems exhibiting unusual forms of magnetic response.

\section{NUMERICAL EXAMPLES}

We now illustrate the above general concept with the help of selected model calculations, intending to highlight typical novel effects. In Fig. 2 we depict quasithermal expectation values (20) for $J=7 / 2, \omega_{0} / \omega=0.1$, and rightcircularly polarized driving (7), as corresponding to the spectrum shown in Fig. 1. In this case the Floquet matrix elements $\left\langle u_{m}(t)\left|S_{z}\right| u_{m}(t)\right\rangle$ do not depend on time [25], eliminating the necessity to compute their temporal averages (21). Here we have set, somewhat arbitrarily, $\gamma_{y}=\gamma_{z}=0$, and consider three spectral bath densities as three prototypical examples for different environments: a constant density $\varrho(\widetilde{\omega})=\varrho_{0}$ (dotted lines), a superohmic quadratic density

$$
\varrho(\widetilde{\omega})=\varrho_{0}(\widetilde{\omega} / \omega)^{2}
$$


(dashed lines), and a Gaussian density

$$
\varrho(\widetilde{\omega})=\varrho_{0} \exp \left[-\left(\widetilde{\omega}-\omega_{\mathrm{c}}\right)^{2} / 2 \omega^{2}\right]
$$

with center frequency $\omega_{\mathrm{c}} / \omega=5.0$ (full lines). For both bath temperatures presupposed in Fig. 2 one encounters a striking manifestation of the nonuniversality of periodic thermodynamics: while all three densities necessarily lead to the same magnetization (4) in ordinary thermodynamics, i.e., for vanishing driving amplitude, namely, $\langle m\rangle_{\mathrm{eq}} \approx-0.52$ for $k_{\mathrm{B}} T /(\hbar \omega)=1.0$ and $\langle m\rangle_{\mathrm{eq}} \approx-2.92$ for $k_{\mathrm{B}} T /(\hbar \omega)=0.1$, the quasithermal expectation values differ distinctly from each other when the drive is turned on. Since positive $\omega_{0}$ correspond to negative $g$ factors, the spin tends to align antiparallely to the applied static field when the oscillating field vanishes, requiring negative expectation values $\langle\langle m\rangle\rangle$ for $F / \omega \rightarrow 0$. Remarkably, in all three cases the sign of the magnetization changes when the oscillating field becomes sufficiently strong [25], as if the sign of $g_{J}$ were reversed through the application of a strong time-periodic drive. The most notable differences enforced by the different environments appear close to the principal resonance, $\Omega / \omega=1$. While the response of a system with constant density of states to the probing field is not particularly affected by this resonance, but vanishes when $\Omega=\omega$, the response of a system with Gaussian density of states exhibits a pronounced dip at this resonance, again vanishing exactly for $\Omega=\omega$. In marked contrast, the superohmic quadratic density of states gives rise to a maximum of the effective mean magnetization when $\Omega=\omega$. These differences can be explained by analyzing the individual factors $\left|V_{f i}^{(\ell)}\right|^{2}$, $N\left(\omega_{f i}^{(\ell)}\right)$, and $\varrho\left(\left|\omega_{f i}^{(\ell)}\right|\right)$, which easily conspire to affect the partial rates (16) in a nonobvious manner, with the density of states serving to enhance or suppress these partial rates for certain $\ell$ [24]. The resonance at $\Omega / \omega=3 / 2$ only reveals itself through sharp spikes in this Fig. 2, whereas no signal can be seen for $\Omega / \omega=2$. We remark that the golden rule-based approach leading to the partial rates (16) might seem endangered close to a resonance, since a vanishing transition frequency (15) entails a diverging expectation value (17). However, the Floquet-state occupation probabilities then are determined by ratios of rates. According to the detailed analysis performed in Ref. [20] these ratios actually remain finite despite the divergence of both numerator and denominator, strongly suggesting that the golden rule does not lose its validity on resonance.

From an experiment-oriented viewpoint it deserves to be pointed out that the maximum absolute value of the quasithermal magnetization deduced from Fig. 2 for comparatively high bath temperature, $k_{\mathrm{B}} T=\hbar \omega=10 \hbar \omega_{0}$, is comparable to the bare equilibrium magnetization occurring at much lower temperature, $k_{\mathrm{B}} T=\hbar \omega / 10=\hbar \omega_{0}$. Hence application of an oscillating field can strongly enhance the magnetization of a paramagnet as corresponding to an effective cooling of the system.

The sign change of the quasithermal magnetization is related to the crossing of all quasienergies observed in Fig. 1 at the principal resonance. In order to further illustrate this connection we also consider a linearly polarized driving field applied orthogonally to the static one,

$$
H_{\mathrm{osc}}^{(l i n)}(t)=\hbar F S_{x} \cos (\omega t) .
$$

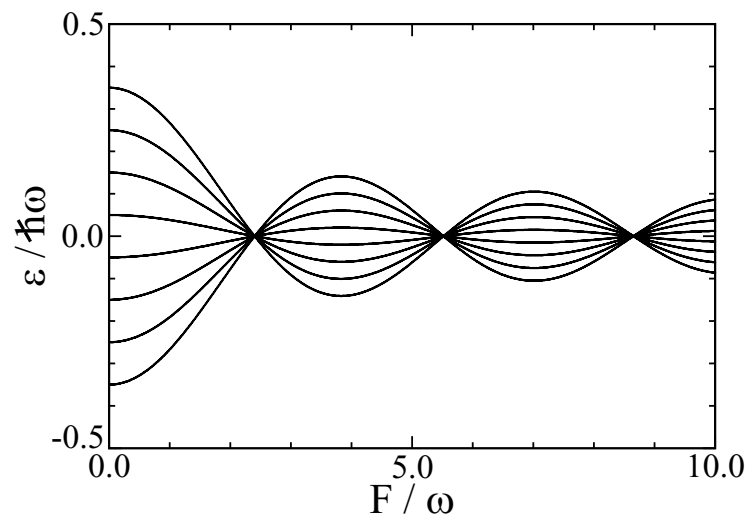

FIG. 3. One Brillouin zone of quasienergies for a linearly driven spin $J=7 / 2$ with $\omega_{0} / \omega=0.1$. Observe how the scale of the abscissa here differs from that in Fig. 1.

The corresponding quasienergy spectrum, again for $J=7 / 2$ and $\omega_{0} / \omega=0.1$, is shown in Fig. 3. This spectrum is described to fair precision by the high-frequency approximation

$$
\varepsilon_{m}=E_{m} J_{0}(F / \omega) \quad \bmod \hbar \omega
$$

known from tight-binding chains with nearest-neighbor coupling under periodic driving [27], with $J_{0}(z)$ denoting the Bessel function of the first kind of order zero. Thus here one finds a sequence of complete quasienergy level crossings at the zeros of $J_{0}$.

Now assuming isotropic coupling $\gamma_{x}=\gamma_{y}=\gamma_{z}$, and employing the same three bath densities $\varrho(\widetilde{\omega})$ as before, the resulting quasithermal expectation values (20) are plotted in Fig. 4. Interestingly, for both temperatures $k_{\mathrm{B}} T /(\hbar \omega)=1.0$ and $k_{\mathrm{B}} T /(\hbar \omega)=0.1$ there is no sign change of the effective magnetization when the density of states is constant, but $\langle\langle m\rangle\rangle$ becomes zero at the quasienergy collapse points observed in Fig. 3. In contrast, both the quadratic density (22) and the Gaussian density (23) give rise to repeated sign changes of the magnetization when the driving amplitude is increased; note that in any case the quasithermal magnetization vanishes at the collapse points.

Further information on the interplay of the quasienergy spectrum and the quasithermal magnetization is obtained when replacing the right-circularly polarized driving field (7) by a left-circularly polarized one, as given by

$$
H_{\mathrm{osc}}^{(l)}(t)=\hbar F\left[S_{x} \cos (\omega t)-S_{y} \sin (\omega t)\right] .
$$

In order to elucidate the underlying symmetry properties, we consider the quasienergy operator for right-circular driving, that is, the operator

$$
K^{(r)}=H_{0}+H_{\mathrm{osc}}^{(r)}(t)-i \hbar \frac{d}{d t},
$$

which determines the corresponding $2 \pi / \omega$-periodic Floquet functions $\left|u_{m}(t)\right\rangle$ and their quasienergies $\varepsilon_{m}$ as solutions of the eigenvalue equation

$$
K^{(r)}\left|u_{m}(t)\right\rangle=\varepsilon_{m}\left|u_{m}(t)\right\rangle
$$



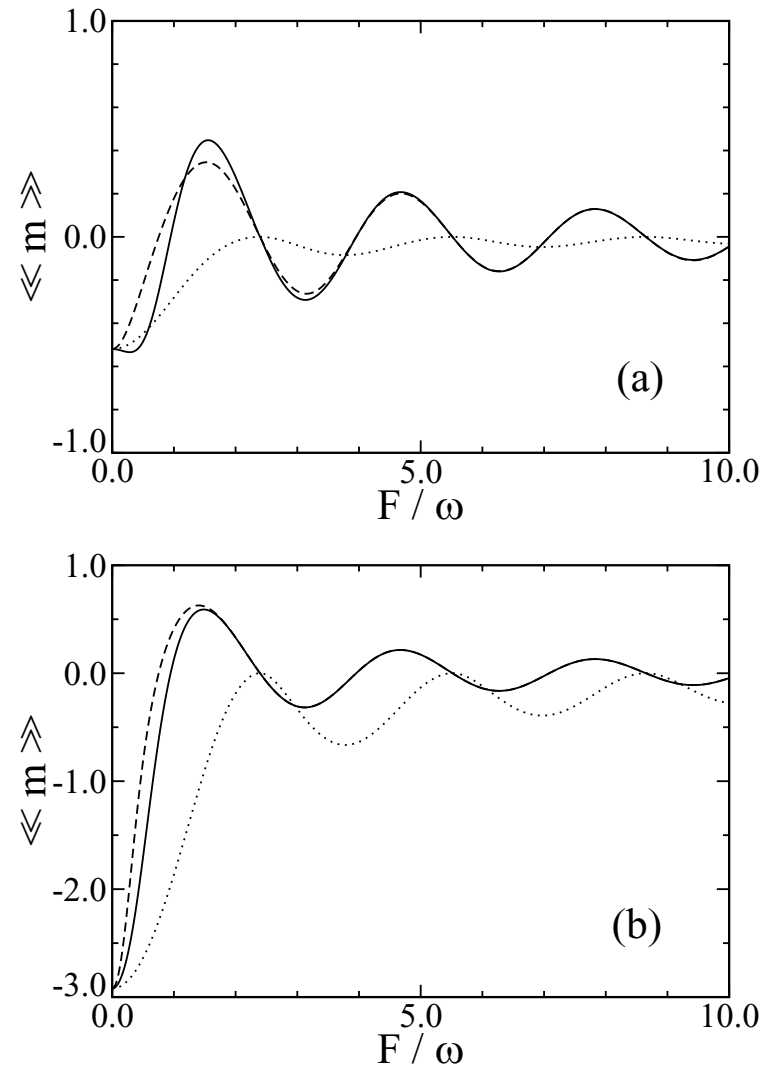

FIG. 4. Quasithermal expectation values (20) for $J=7 / 2$, $\omega_{0} / \omega=0.1$, and linearly polarized driving described by Eq. (24). Here $\gamma_{x}=\gamma_{y}=\gamma_{z}$; the scaled bath temperature is $k_{\mathrm{B}} T /(\hbar \omega)=1.0$ in the upper panel (a) and $k_{\mathrm{B}} T /(\hbar \omega)=0.1$ in the lower one (b). The bath density of states is taken to be constant (dotted), quadratic (dashed) according to Eq. (22), and Gaussian (full lines) with $\omega_{\mathrm{c}} / \omega=5.0$; see Eq. (23).

and subject this operator (27) to the spatiotemporal parity operation

$$
\mathcal{P} T:\left\{\begin{array}{l}
\vec{S} \rightarrow-\vec{S}, \\
t \rightarrow-t,
\end{array}\right.
$$

simultaneously reversing the sign of the components $S_{x}, S_{y}, S_{z}$ of the spin vector $\vec{S}$, and that of the time coordinate $t$. Under this operation, one has

$$
K^{(r)} \rightarrow-K^{(l)},
$$

where $K^{(l)}$ is the quasienergy operator for left-circular driving, being obtained from $K^{(r)}$ through the replacement of $H_{\mathrm{osc}}^{(r)}(t)$ by its left-handed counterpart (26). Now the operation (29) can be separated into two consecutive steps: (i) a sign change of $S_{z}$ alone, as corresponding to a reversal of the direction of the static field $B_{0}$, and (ii) the combined remaining sign changes of $S_{x}, S_{y}$, and $t$, as corresponding to a transformation of $H_{\text {osc }}^{(r)}(t)$ into $-H_{\text {osc }}^{(l)}(t)$. Since the sign of $H_{\text {osc }}(t)$ does not affect the quasienergy spectrum in any case, and $-K^{(l)}$ is isospectral to $K^{(l)}$, this means that the quasienergy spectrum for left-circular driving with positive $\omega_{0}$ is the same as that for right-circular driving with negative $\omega_{0}$, and therefore again is given by Eq. (9). This is intuitively clear, since changing the

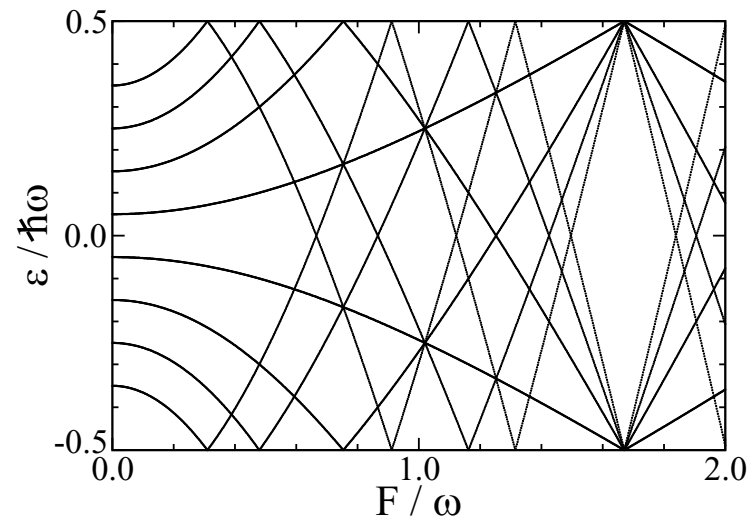

FIG. 5. One Brillouin zone of quasienergies for a spin $J=7 / 2$ driven by a left-circularly polarized high-frequency magnetic field according to Eq. (26), and exposed to a static magnetic field of scaled strength $\omega_{0} / \omega=0.1$. High degeneracies are found at $F / \omega \approx 1.02$ $(\Omega / \omega=3 / 2)$ and $F / \omega \approx 1.67(\Omega / \omega=2)$.

"handedness" of the circularly polarized drive should have the same effect as a reversal of the direction of the orthogonally applied static field. By analogous reasoning, the quasithermal magnetization for left-circular driving with positive $\omega_{0}$ is the negative of that for right-circular driving with negative $\omega_{0}$. But with $\omega \gg-\omega_{0}>0$, the canonical representatives of the quasienergies (9) repel each other with increasing driving strength, as shown in Fig. 5, so that there is no quasienergy collapse. As a consequence, the quasithermal magnetization induced by a left-circularly polarized driving field is substantially different from that due to a right-circular drive, as exemplified by Fig. 6 for the same conditions previously considered in Fig. 2, except for the handedness of polarization. As expected, there is no sign change of the quasithermal magnetization plotted in Fig. 6, reflecting the absence of the principal resonance. However, for moderate driving amplitudes a noteworthy increase of the magnetization is achieved, reflecting "Floquet-state cooling by driving" [24]. This effect is particularly visible for $k_{\mathrm{B}} T /(\hbar \omega)=1.0$ and the Gaussian density of states; in that case the maximum quasithermal magnetization exceeds the equilibrium magnetization by a factor of more than five.

\section{EXPERIMENTUM CRUCIS}

Periodic thermodynamics of driven quantum systems, as envisioned by Kohn [7], constitutes an area somewhere in between equilibrium thermodynamics on the one hand and nonequilibrium thermodynamics of more general systems on the other. The fact that such periodically driven systems possess a basis of states - the Floquet states - which are occupied according to certain distributions $\left\{p_{n}\right\}$ when being in contact with a heat bath is a feature they share with equilibrium systems. The fact that these distributions are not universal, but do depend on the very details of the system-bath interaction, distinguishes them sharply from equilibrium systems, and can be exploited for deliberate quantum engineering.

The present model study has identified consequences of periodic thermodynamics for ideal paramagnetic or diamagnetic 

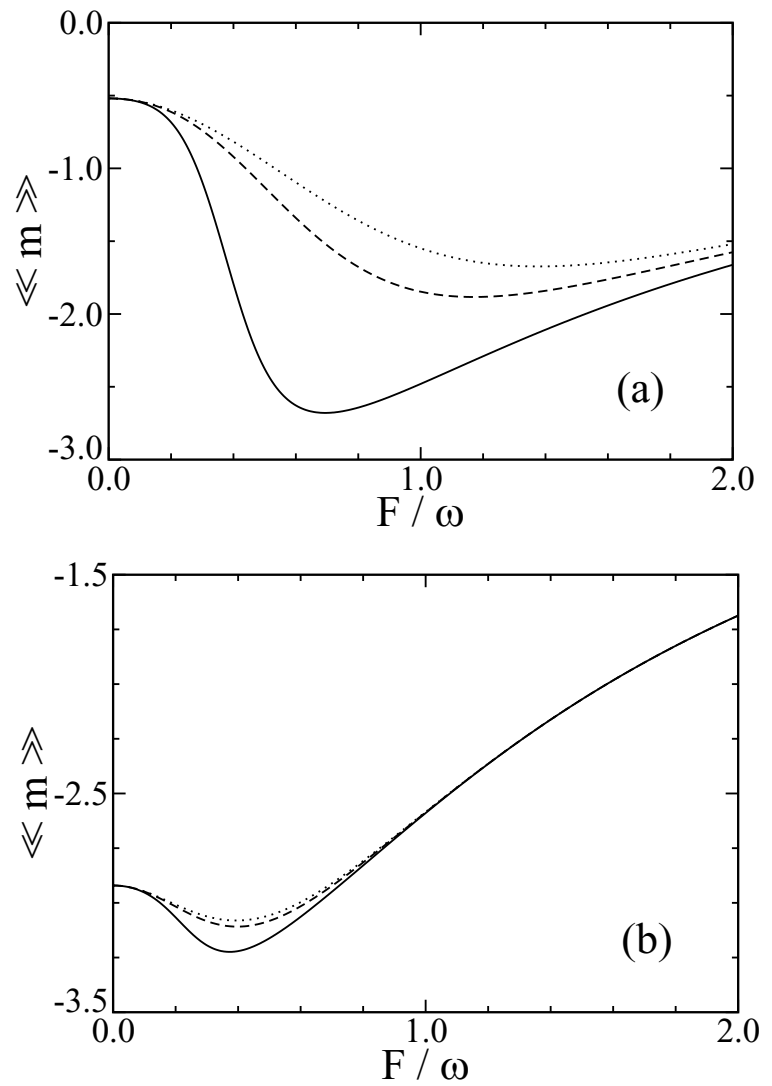

FIG. 6. As Fig. 2, but for left-circularly polarized driving described by Eq. (26). Observe that the quasithermal magnetization is enhanced, so that the system is effectively cooled through the application of moderately strong fields.

spin systems in contact with a thermal environment, which are well amenable to accurate experimental investigation ever since Henry's seminal measurements [3]. The "quasithermal" magnetization exhibited by such systems under the influence of a strong oscillating magnetic field shows at least four characteristic signatures.

(i) Different paramagnetic materials which respond in precisely the same universal way to a static magnetic field in thermal equilibrium [1,2] may respond differently in the presence of an additional strong oscillating field, depending on how the spins interact with their environment.

(ii) In the case of a circularly polarized driving field, quasithermal magnetization is strongly sensitive to the former's handedness.

(iii) The quasithermal magnetization in the presence of periodic driving can be even larger than the equilibrium thermal magnetization in the absence of the drive, as if the system was effectively cooled [24].

(iv) Even the sign of the quasithermal magnetization can be different from that of the equilibrium thermal magnetization [25], reflecting a quasienergy collapse.

Verification of these signatures constitutes an experimentum crucis for periodic thermodynamics: they are so elemental that they simply have to exist if the underlying theoretical framework is correct.

Extensive experimental measurements of quasithermal magnetization will not only be of conceptual merit, but also provide information not available otherwise, because quasithermal magnetic response encodes the details of the mechanism by which the driven spins are interacting with their surroundings. While these details necessarily have to remain hidden in measurements of ordinary thermal magnetism, due to the censorship imposed by the universality of equilibrium thermodynamics, the nonuniversality of periodic thermodynamics has the power to lift this censorship, and to make them visible.

\section{ACKNOWLEDGMENTS}

This work has been supported by the Deutsche Forschungsgemeinschaft (DFG, German Research Foundation) through Project No. 397122187. We wish to thank the members of the Research Unit FOR 2692 for many stimulating discussions.
[1] L. Brillouin, Les moments de rotation et le magnétisme dans la mécanique ondulatoire, J. Phys. Radium 8, 74 (1927).

[2] For a modern exposition see, e.g., R. K. Pathria and P. D. Beale, Statistical Mechanics, 3rd ed. (Academic Press, New York, 2011).

[3] W. E. Henry, Spin paramagnetism of $\mathrm{Cr}^{+++}, \mathrm{Fe}^{+++}$, and $\mathrm{Gd}^{+++}$ at liquid helium temperatures and in strong magnetic fields, Phys. Rev. 88, 559 (1952).

[4] M. Bałanda, AC susceptibility studies of phase transitions and magnetic relaxation: Conventional, molecular and lowdimensional magnets, Acta Phys. Pol. A 124, 964 (2013).

[5] I. Klik, J. McHugh, R. W. Chantrell, and C.-R. Chang, Debye formulas for a relaxing system with memory, Sci. Rep. 8, 3271 (2018).

[6] C. V. Topping and S. J. Blundell, A. C. susceptibility as a probe of low-frequency magnetic dynamics, J. Phys.: Condens. Matter 31, 013001 (2019).
[7] W. Kohn, Periodic thermodynamics, J. Stat. Phys. 103, 417 (2001).

[8] R. Graham and R. Hübner, Generalized quasi-energies and Floquet states for a dissipative system, Ann. Phys. (NY) 234, 300 (1994).

[9] H.-P. Breuer and F. Petruccione, Dissipative quantum systems in strong laser fields: Stochastic wave-function method and Floquet theory, Phys. Rev. A 55, 3101 (1997).

[10] D. W. Hone, R. Ketzmerick, and W. Kohn, Statistical mechanics of Floquet systems: The pervasive problem of near degeneracies, Phys. Rev. E 79, 051129 (2009).

[11] R. Ketzmerick and W. Wustmann, Statistical mechanics of Floquet systems with regular and chaotic states, Phys. Rev. E 82, 021114 (2010).

[12] S. Gasparinetti, P. Solinas, S. Pugnetti, R. Fazio, and J. P. Pekola, Environment-Governed Dynamics in Driven Quantum Systems, Phys. Rev. Lett. 110, 150403 (2013). 
[13] M. Langemeyer and M. Holthaus, Energy flow in periodic thermodynamics, Phys. Rev. E 89, 012101 (2014).

[14] G. Bulnes Cuetara, A. Engel, and M. Esposito, Stochastic thermodynamics of rapidly driven systems, New J. Phys. 17, 055002 (2015).

[15] T. Shirai, T. Mori, and S. Miyashita, Condition for emergence of the Floquet-Gibbs state in periodically driven open systems, Phys. Rev. E 91, 030101(R) (2015).

[16] S. Restrepo, J. Cerrillo, V. M. Bastidas, D. G. Angelakis, and T. Brandes, Driven Open Quantum Systems and Floquet Stroboscopic Dynamics, Phys. Rev. Lett. 117, 250401 (2016).

[17] M. Hartmann, D. Poletti, M. Ivanchenko, S. Denisov, and P. Hänggi, Asymptotic Floquet states of open quantum systems: The role of interaction, New J. Phys. 19, 083011 (2017).

[18] J. Zhang, P. W. Hess, A. Kyprianidis, P. Becker, A. Lee, J. Smith, G. Pagano, I.-D. Potirniche, A. C. Potter, A. Vishwanath, N. Y. Yao, and C. Monroe, Observation of a discrete time crystal, Nature (London) 543, 217 (2017).

[19] S. Choi, J. Choi, R. Landig, G. Kucsko, H. Zhou, J. Isoya, F. Jelezko, S. Onoda, H. Sumiya, V. Khemani, C. von Keyserlingk, N. Y. Yao, E. Demler, and M. D. Lukin, Observation of discrete time-crystalline order in a disordered dipolar many-body system, Nature (London) 543, 221 (2017).

[20] H.-J. Schmidt, Periodic thermodynamics of a two spin Rabi model, J. Stat. Mech. (2020) 043204.

[21] T. N. Ikeda and M. Sato, General description for nonequilibrium steady states in periodically driven dissipative quantum systems, arXiv:2003.02876 [cond-mat.stat-mech].

[22] H.-P. Breuer, W. Huber, and F. Petruccione, Quasistationary distributions of dissipative nonlinear quantum oscillators in strong periodic driving fields, Phys. Rev. E 61, 4883 (2000).

[23] O. R. Diermann, H. Frerichs, and M. Holthaus, Periodic thermodynamics of the parametrically driven harmonic oscillator, Phys. Rev. E 100, 012102 (2019).

[24] O. R. Diermann and M. Holthaus, Floquet-state cooling, Sci. Rep. 9, 17614 (2019).

[25] H.-J. Schmidt, J. Schnack, and M. Holthaus, Periodic thermodynamics of the Rabi model with circular polarization for arbitrary spin quantum numbers, Phys. Rev. E 100, 042141 (2019).

[26] H.-P. Breuer and F. Petruccione, The Theory of Open Quantum Systems (Oxford University Press, Oxford, 2002).

[27] M. Holthaus, The quantum theory of an ideal superlattice responding to far-infrared laser radiation, Z. Phys. B 89, 251 (1992). 